\title{
Moral Assertion
}

\section{Christoph Kelp ${ }^{1}$ (I)}

Accepted: 14 July 2020 / Published online: 15 August 2020

(C) The Author(s) 2020

\begin{abstract}
In this paper, I introduce a puzzle about moral assertion and defend a solution that centrally features the claim that the normativity of moral assertion centrally features moral understanding.
\end{abstract}

Keywords Moral assertion $\cdot$ Norms of assertion $\cdot$ Moral knowledge $\cdot$ Moral understanding

1. A knowledgeable and reliable informant, A, comes up to you and tells you that tonight's Champions League match will be screened at the local pub. Based on this testimony, you form the corresponding belief and subsequently spread the word to B.

What we have here is a default case of testimony: A approaches you and tells you a humdrum truth. It is also a default case of knowledge transmission via testimony, or so we may at any rate assume. A knows what she tells you and when you form the corresponding belief based on her assertion, you come to know what you are being told as well. Finally, the case is a default case of you telling B what you know. All of this is perfectly familiar and entirely unproblematic.

Consider next a variation of the case in which the only thing that changes is the content of what you are being told. Rather than a humdrum truth A offers you a moral truth, say that it's immoral to patronise the local pub. Again, based on this testimony, you form the corresponding belief and subsequently spread the word to B. We have another default case of testimony, transmission of knowledge by testimony and asserting what you know. ${ }^{1}$

Since we have two default cases of testimony, transmission of knowledge by testimony and asserting what you know and since the only difference between the two cases resides in the content of what you are being told, we may expect that what's true in one case is true in the other also. Crucially, however, that's not what we find. In fact, there is an important difference between the two cases. While in the non-moral case, it is perfectly fine to assert your testimonial belief to B in the way you do, the same does not hold for the moral case. On the

${ }^{1}$ I have been encouraged to state that I am assuming a cognitivist view of moral assertion.

Christoph Kelp

christoph.kelp@glasgow.ac.uk

1 Philosophy, University of Glasgow, 69 Oakfield Ave, Glasgow G12 8LP, UK 
contrary, asserting your testimonial belief to B is on the face of it rather problematic. That's surprising, if not mysterious.

In fact, what the moral assertion case shows is that the following three individually plausible claims cannot all be true:

\section{The Moral Assertion Puzzle}

MAP 1 In default cases, assertions from knowledge are proper. ${ }^{2}$

MAP 2 In default cases, assertions from knowledge are a source of testimonial knowledge. ${ }^{3}$

MAP 3 In default cases, moral assertions in the light only of moral testimony are not proper.

Here is how the moral assertion case shows MAP1-3 to be inconsistent. First, it's a default case of (moral) assertion from knowledge: A comes up to you and tells you something A knows, in this case a moral truth. Since by MAP2, assertions from knowledge are a source of testimonial knowledge in default cases, we may assume that A's moral assertion that it's immoral to patronise the local pub generates the corresponding knowledge in you. When you go on to spread the word to B, we are yet again dealing with a default case of moral assertion from knowledge. By MAP1, your moral assertion will be entirely in order, too. Since the belief you assert is based only on moral testimony (from A), by MAP3, the moral assertion you make in light of this knowledge is not proper. But since it cannot be that your assertion in light of your testimonial moral knowledge both is and is not proper, one out of MAP1-3 must be false. ${ }^{4}$

2. But which one? First, MAP3 carries little promise. After all, it receives strong support by the clear intuitive difference between the non-moral case in which your assertion in the light only of testimony is proper, intuitively, and the moral case in which it isn't. This intuitive difference requires an explanation. And, on the face of it at least, the best explanation of this intuitive difference is that in the non-moral case, it is true that your assertion in light only of testimony is proper, whereas in the moral case, it isn't. In this way, MAP3 receives strong intuitive support.

What about MAP2? While some have indeed argued that testimony is not a source of moral knowledge (e.g. Wolff 1970), there is excellent reason for thinking that, at the end of the day, this isn't the way to go either. Consider a piece of moral knowledge that you have, perhaps even that it is immoral to patronise the local pub. Of course, we must now assume that you did not acquire this piece of moral knowledge from testimony. Instead, let's assume that you acquired it based on inference from the fact that the local pub is using small children to wash their dishes and that is why it is immoral to patronise the pub.

\footnotetext{
${ }^{2}$ The related knowledge norm of assertion is the most popular view on the norm of assertion. For defences see e.g. (Kelp 2018; Benton 2011; Hawthorne 2004; Simion 2016; Turri 2011; Unger 1975; Williamson 2000).

${ }^{3}$ The related non-reductionism about testimonial knowledge is the most popular view on testimonial knowledge. For defences see e.g. (Simion and Kelp 2020; Burge 1993; Coady 1992; Goldman 1999; Goldberg 2006).

${ }^{4}$ That testimony has puzzling consequences in the moral domain is fairly widely recognised e.g. (Hills 2009; McGrath 2011). Typically, this puzzle is viewed as concerning the oddity of trusting moral testimony. While there may well be a puzzle about trusting moral testimony, note that the Moral Assertion Puzzle is different. To see this, note first that the only plausible way to convert the above puzzle into a puzzle about trusting testimony is by rejecting MAP2 (in a certain way). At the same time, however, there is reason to think that this would be a mistake, as I will argue in due course. Moreover, so long as we have reason to hold on to MAP2 and moral assertion is a source of moral knowledge at least in default cases, the Moral Assertion Puzzle remains no matter whether there is also something odd about trusting testimony. While there is reason to think that any puzzle about trusting moral testimony is different from the Moral Assertion Puzzle, one may still wonder whether the two puzzles share a common source. This is a fascinating question. However, one can only do so much in any one paper. That is why I will not pursue it any further here.
} 
Suppose that you find that when you talk to your friends about this, they agree that it is immoral to patronise an establishment that uses small children to wash their dishes. It is hard to deny that you can now be even more confident that it is immoral to patronise the local pub. But given that this is so, your friends' assertions must generate justification for you. Otherwise, it would not be permissible for you to increase your confidence in the proposition under consideration. So, there is no in principle obstacle to assertion generating moral justification for you. But if there is no in principle obstacle, it is hard to see why moral assertion shouldn't generate moral justification in default cases like the above as well. Finally, if moral assertion does generate moral justification, there is no in principle obstacle to moral assertion generating moral knowledge either. ${ }^{5}$ After all, we may assume that you acquire the corresponding belief, that your belief is true and that you are not gettiered. As a result, there is reason to think that MAP2 stands strong as well.

3. Of course, if MAP2 and 3 are both true, then MAP1 must be the culprit. Moral knowledge is not enough for moral assertion to be proper. This raises the question as to what is enough. My suggestion is that the normativity of moral assertion centrally features understanding (and explanation). In this way, it differs from the normativity of non-moral assertion, which centrally features knowledge. In the remainder of this paper, I will argue that this solution is plausible on independent grounds ${ }^{6}$

Let me first rehearse the relevant argument that the normativity of non-moral assertion centrally features knowledge (cf. Kelp 2018). Since it is a theoretical argument, I will first have to do a bit of stage setting, starting with some key conceptual machinery. First, we need to understand etiological functions (e-functions). An etiological function is defined as follows:

\section{Etiological Function}

\section{A token of type $\mathrm{T}$ has the e-function of producing effect $\mathrm{E}$ in system $\mathrm{S}$ if and only if}

\section{(EF1) Past tokens of T produced E in S's ancestors ${ }^{7}$}

\footnotetext{
${ }^{5}$ It might be thought that this is too quick. This is because it might be thought that moral assertion will generate moral justification that's strong enough for moral knowledge that $\mathrm{p}$ only if it also generates moral understanding why p. If so, MAP1 affords an important restriction. What's more, this restriction promises to solve the Moral Assertion Puzzle. To see this, note that in our toy case, moral assertion does not generate moral understanding. According to the present proposal, then, it does not generate moral knowledge either. The asymmetry between the moral and the non-moral case is duly explained. Unfortunately, there is reason to think that an understanding requirement on testimonial moral knowledge is just too strong. Suppose you are on the phone with me, talking about a common acquaintance, X. I say: "You won't believe what X did today. It was so immoral. He ..." At this point the line is cut. It is clear that you acquire moral knowledge based on my testimony, i.e. that X did something immoral today, but not the corresponding moral understanding why. For further critical discussion see e.g. (Hills 2009; McGrath 2011).

${ }^{6}$ Note the most obvious alternative candidates, which analyse the normativity of moral assertion in terms of higher-order knowledge or certainty, won't do the trick. To see this, suppose that, in the above case, your testifier who tells you that it is immoral to patronise the local pub is god or an oracle that is commonly known to be infallible. In that case you have higher-order knowledge and even certainty that it is immoral to patronise the local pub. At the same time, it would still be problematic for you to go and spread the word.

A related idea that is fairly widely defended in the literature is the idea that morally worthy action requires moral understanding e.g. (Hills 2009; Nickel 2001). Note, however, that this view differs from the present one in that the understanding norm on morally worthy action here is a moral norm, whereas the understanding norm of moral assertion is an epistemic norm (as will become clear in due course).

${ }^{7}$ It may be worth pointing out that an ancestor can also be an earlier cycle of the system (Graham 2014).
} 
(EF2) Producing E benefitted S's ancestors

(EF3) Producing E's having benefitted S's ancestors explains why T continues to exist in S.

The paradigm case of an e-functional type is the human heart. Its e-function consists in pumping blood (Graham 2012). This is because past hearts pumped blood in our ancestors (EF1); this benefitted our ancestors (by keeping them alive, EF2); and the fact that pumping blood benefitted our ancestors explains why the heart continues to exist (by explaining the proliferation of the genes responsible for the heart, EF3).

To fully understand what e-functions are, we will also need to understand the notions of function fulfilment, normal functioning and normal conditions. A token of a functional type fulfils its e-function if and only if it produces the e-functional effect. In the case of the heart, this is the pumping of blood. Normal conditions are the conditions that obtained when the type acquired the relevant e-function. In the case of the heart, this involves being hooked up to arteries and veins in a certain way. And, finally, normal functioning is the way tokens of the type functioned when the type acquired its function. In the case of the heart, this involves beating at a certain rate.

Finally, e-functions can be typed by the type of benefit they produced in the system. For instance, the function of pumping blood is a biological e-function of the heart because the benefit of keeping us alive is a biological benefit (Kelp 2018; Simion 2016).

4. Crucially for present purposes, e-functions have normative import. First, for functional types, there is a fact of the matter as to whether a token of the type is a good token of the type. In particular, a token of a functional type is good if and only if it has the disposition to fulfil its e-function when functioning normally ${ }^{9}$ in normal conditions (Kelp 2018). For instance, a good heart is a heart that has the disposition to pump blood by beating at a certain rate when hooked up to arteries and veins in a certain way. Let's call this the evaluative norm of the functional type. Second, there is a fact of the matter as to how various parts of the system relative to which a given efunction is defined ought to function. In particular, a part of the system functions as it ought to if and only if it is functioning normally (Graham 2012). For instance, a heart functions as it ought to when it beats a certain rate. Let's call this the prescriptive norm of the part of the system. Finally, norms can be typed in accordance with the type of function, i.e., ultimately, by the relevant type of benefit. The abovementioned norms governing the heart thus come out as biological norms.

5. Let's move on to another key idea, which is the idea of certain kind of simple economic system (SES). An SES is constituted by a type of producer, a type of product, a type of consumer and a type of return and such that the producer produces tokens of the product which can be consumed by consumers in exchange for a return (Kelp 2018).

We are all familiar with SESs. Your coffee dealer and you may constitute an SES. Your coffee dealer (producer) produces coffees (product) which you (consumer) may consume in exchange for a return (money). Crucially, products in SESs often have e-functions. In the SES under consideration, the coffee your coffee dealer produces may well have the e-function of producing pleasant gustatory experiences in you. That's something past servings of coffee

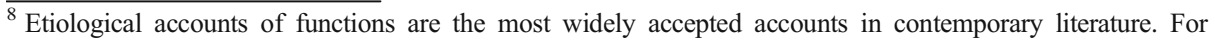
prominent defences see (Millikan 1984; Neander 1991; Griffith 1993; Godfrey-Smith 1994; Buller 1998). For applications of e-functions in epistemology see (Kelp 2018; Graham 2012, 2014; Simion 2019).

${ }^{9}$ Note that it is the tokens that need to be functioning normally, not the entire system. The reasons for this will become clear in due course.
} 
have done (EF1), it is something that benefits you (EF2), and the fact that it benefits explains why the product type continues to exist in the system (EF3). If the last claim isn't immediately obvious, note that the fact that you liked the coffee motivated you to come back and pay for more coffee. And that motivated your coffee dealer to continue producing the coffee, which is why the product type continues to exist in the system.

6. Crucially for present purposes, the practice of making and consuming assertions is an SES. Speakers are producers, hearers are consumers, assertions are the product and belief on the part of the hearer is the return (Kelp 2018; Graham 2010; Millikan 2004). Assertion has the e-function of generating knowledge in hearers. This is because past assertions have done so (EF1), this was beneficial for hearers (EF2) and the fact that it was beneficial for hearers explains why assertion continues to exist in the system (EF3). To see why, note that the fact that assertion generates knowledge in hearers explains why assertions continue to be consumed by hearers and the fact that hearers continue to respond to assertion with belief explains why speakers continue to produce them. Finally, since the benefit of generating knowledge in hearers is an epistemic benefit, the e-function of generating knowledge in hearers is an epistemic function of assertion (Kelp 2018).

7. Since assertion has the e-function of generating knowledge in hearers, we can reap the normative benefits of e-functions. In particular, the results that we get are (i) an evaluative norm according to which a good assertion is one that has the disposition to generate knowledge in hearers when functioning normally in normal conditions; and (ii) prescriptive norms according to which various parts of the SES of producing and consuming assertions function as they ought if and only if they are functioning normally. And, of course, since the relevant function generating these norms is an epistemic function, the norms are epistemic norms.

What does normal functioning in the practice of producing and consuming assertions amount to? It is very plausible that it involves at least the following: a speaker asserts that $p$ and a hearer comes to believe that $\mathrm{p}$ based on the speaker's assertion that $\mathrm{p}$. Moreover, normal functioning on the speaker's side involves asserting from knowledge. This gives us the familiar prescriptive norm of assertion: a speaker asserts as they ought to if and only if they assert from knowledge. Moreover, this norm comes out as a distinctively epistemic norm.

8. Of course, the lesson the moral assertion puzzle teaches us is that there is reason to believe that knowledge isn't enough for proper moral assertion. The reason for this, I want to suggest, is that moral assertion has a stronger e-function. More specifically, it has the efunction of generating moral understanding in hearers. ${ }^{10}$

To see why the function cannot just be knowledge, note that although past moral assertions may well have produced moral knowledge in our ancestors (EF1), and it may well have been that this was beneficial for them, at least to some extent (EF2), it is just not very plausible that it was this benefit that explains the continued existence of moral assertion. If this isn't immediately obvious, note that we would typically not expect hearers to respond to assertions of moral fact without a backing explanation (henceforth also 'unexplained moral assertion') with belief. For instance, we would typically not expect hearers to respond to your assertion that it is immoral to patronise the local pub with belief; not unless you also offer some reason

\footnotetext{
${ }^{10}$ What exactly does it take to have moral understanding? In my view, understanding is roughly systematic knowledge (Kelp 2015) and moral understanding is, again roughly, systematic moral knowledge (Kelp 2020, unpublished). (Note that while this is a knowledge-based account of moral understanding, moral understanding does not reduce to knowledge. This is because it also needs to be systematic. That's why the account is not committed to MAP1.) For alternative views of moral understanding, see e.g. (Callahan 2018; Hills 2009; Howard 2018; Sliwa 2017).
} 
why this is so. I, for starters, most certainly wouldn't (no offense). But, of course, if hearers do not respond with belief to unexplained moral assertion, speakers will not get the desired return for their assertion. Without the desired return, speakers will lose motivation to continue to make moral assertions with the result that the practice of producing and consuming moral assertions will disappear, at least in the long run. ${ }^{11}$

It is no surprise that unexplained moral assertions are a rare occurrence in the practice of making and consuming moral assertions. Much more common are what I will call 'explained moral assertions', which come with a backing explanation of the relevant moral fact. For instance, a much more familiar way for our toy case to proceed is as follows. Your reliable informant tells you that it is immoral to patronise the local pub because they employ small children to wash dishes. When this happens, typically, you not only come to know that it is immoral to patronise the local pub, but you also come to understand why it is immoral. Most importantly for present purposes, it is plausible that generating moral understanding why is an e-function of moral assertion. It is something that past moral assertions did (EF1), it was beneficial for hearers (EF2) and it plausibly explains why moral assertion continues to exist (EF3). This is because it is plausible that, in case of explained moral assertion, hearers do respond with belief, thus offering the desired return that will motivate speakers to continue to produce moral assertions. Were you to tell me that it is immoral to patronise the local pub because they employ small children to wash their dishes, I, at least, would respond with belief. Note, finally, that since the benefit of acquiring moral understanding why is an epistemic benefit, the e-function of generating moral understanding is an epistemic e-function of moral assertion. ${ }^{12}$

9. If moral assertion does have the e-function of generating moral understanding in hearers, we get the familiar normative benefits. In particular, in much the same way as for non-moral assertion, we get (i) an evaluative norm of assertion according to which a good moral assertion is one that has the disposition to generate understanding in hearers when functioning normally in normal conditions; and (ii) prescriptive norms for various parts of the SES of producing and consuming moral assertions according to which moral assertions function as they ought if and only if they are functioning normally. And since the relevant function generating these norms is an epistemic function, the norms turn out to be epistemic norms.

What does normal functioning in the practice of producing and consuming moral assertions amount to? It is very plausible that it is at least the following: a speaker offers an explained moral assertion from moral understanding why and a hearer forms the corresponding moral beliefs based on the speaker's moral assertion. Just as in the non-moral case, what's key here is what normal functioning on the speaker's side amounts to. Crucially, unlike in the case of nonmoral assertion where it involved asserting from knowledge, in the moral case, it involves asserting from moral understanding. Accordingly, what we get is the following prescriptive epistemic norm of moral understanding: a speaker morally asserts as they ought to if and only if their moral assertion derives from moral understanding. ${ }^{13}$

\footnotetext{
${ }^{11}$ Note that this is not to say that unexplained moral assertions don't give speakers justification for believing their contents and that as a result it's impermissible for hearers to form the beliefs. On the contrary, the considerations in support of MAP2 from Section 2 suggest that they do. Rather, what's going on here is that in moral assertion cases hearers want more than just justified belief.

${ }^{12}$ Compatibly with this, generating moral understanding might be beneficial in other respects as well. If so, generating moral understanding may be an e-function of assertion of another type as well.

${ }^{13}$ Though see below for a slight refinement and see Turri (2015) for a defence of the more general claim that understanding is the norm of explanation (in fact, on the plausible assumption that explanations have the function of generating understanding, the above can be generalised to provide a theoretical argument for Turri's view). It has been brought to my attention that since I first submitted the paper to this journal (in February 2019), Max Lewis (2020) has published a paper that also defends an understanding norm of moral assertion.
} 
10. I'd now like to consider an objection to this view from a recent paper by Mona Simion (2018). Simion also argues that a central function of moral assertion consists in generating moral understanding in hearers. However, the norm on moral assertion she defends is slightly different than the understanding norm countenanced above. Here goes:

\section{Explanation Proffering}

One's moral assertion that $\mathrm{p}$ is epistemically permissible only if (1) one knows that $\mathrm{p}$ and

(2) one's assertion is accompanied by an appropriate explanation why p. (Simion 2018, 483).

Crucially, according to Simion, Explanation Proffering is preferable to the understanding why norm. This is because it is more closely associated with reliably generating understanding in hearers. Reliably generating understanding in hearers doesn't require understanding on the part of the speaker. According to Simion, this is because the speaker may not satisfy the key 'grasping' condition on understanding that is widely countenanced and so may fail to attain moral understanding why herself. Compatibly with that, when satisfying Explanation Proffering, her moral assertions will reliably generate understanding in hearers provided they satisfy Explanation Proffering. And, conversely, Simion argues that understanding why on the part of the speaker isn't enough for reliably generating understanding in hearers: the speaker may be bad at explaining and so fail to generate understanding in hearers even though she has understanding herself (Simion 2018, 486).

So, shouldn't we go for Explanation Proffering rather than the understanding norm of assertion that I developed above? No. And here is why. Note, first, that Simion's sufficiency worry does not arise for the present proposal. This is because normal functioning in the practice of producing and consuming moral assertions involves not only asserting from moral understanding but also offering explained moral assertions. To live up to the norm generated by normal functioning in the practice, speakers must not only possess understanding why, they must also offer explained moral assertions. In this way, Simion's sufficiency worry can be laid to rest.

What about the necessity worry? I agree that Simion is right to point out that moral understanding on the part of the speaker is not needed to generate understanding why on the part of the hearer, not even to generate it reliably. If what fixes the norm of moral assertion is what's minimally needed for reliably generating moral understanding in hearers, the speaker does not need to have the relevant moral understanding. At the same time, if the norm of moral assertion is fixed in this way, there is excellent reason to believe, pace Simion, that knowledge that $\mathrm{p}$ is not needed for proper moral assertion either. To see why not, note that selfless moral assertions will reliably generate moral understanding in hearers even though the speaker does not know what she asserts. Consider, for instance, a case featuring a closet moral nihilist who also thinks that non-nihilism is best supported by the evidence and has adopted a policy of asserting in accordance with the evidence. As a true nihilist, she does not believe and hence does not know moral truths. Even so, when she asserts them and offers relevant explanations, she will just as reliably generate moral understanding in her audience as her closest non-nihilist counterpart. ${ }^{14}$ If so, knowledge that $\mathrm{p}$ should also not be required for permissible moral assertion by Simion.

${ }^{14}$ Cases of selfless assertion are due to Jennifer Lackey (2008). The above case is an adaptation of Lackey's famous case of the creationist teacher. 
One might wonder how this helps. All it shows is that even Simion's Explanation Proffering, which is weaker than the understanding norm I have offered, is too strong. Crucially, however, I think that the problem does not lie with Simion's knowledge requirement on the norm of moral assertion, but with the way she justifies the content of the norm, i.e. in accordance with what is minimally needed to generate moral understanding in hearers reliably. There are two reasons for this.

First, once we have established that moral assertion has the e-function of generating moral understanding in hearers, there is no further need to provide an independent justification of the relevant norms. After all, we can already derive relevant norms from this e-function and facts about normal functioning and normal conditions.

Second, and more importantly, norms that are generated by e-functions need not and often will not feature the minimal condition that reliably generates the e-functional effect. A certain bird's wing may have the e-function of enabling it to fly. This serves to generate a norm for how the wing should be structured, i.e. in accordance with the way it was structured when the function was acquired. Compatibly with that, it may well be that the bird's wing would enable it to fly with equal reliability, even if some of its bones were missing, provided that the remaining bones were suitably related in a different way. What matters to how e-functions generate norms is that certain properties have actually clustered together in a way that produced the relevant e-functional effect. In the case of nonmoral assertion, these properties are, of course, belief, assertion and knowledge. Given that we normally assert what we believe, it makes sense that normally functioning assertion that generates knowledge involves assertion from knowledge. The fact that belief can be bypassed in a way that assertion still reliably generates knowledge is of little consequence. In the case of moral assertion, they are belief, assertion, moral understanding and moral explanation. Given that we normally assert what we believe and given that we can normally explain what we understand, it makes sense that normally functioning moral assertion that generates understanding involves moral explanation and is made from moral understanding. The fact that we can bypass moral understanding is of equally little consequence.

11. Earlier I said that I take the problem with Simion's argument not to reside in her knowledge requirement as being too strong but in the way she justifies the content of her norm. Even if I am right that there is a problem with Simion's justification, one might wonder why it should be thought that there isn't also a problem with the knowledge requirement. To see why, note that selfless moral assertions are intuitively fine. As a result, cases of selfless moral assertion (e.g. by the moral nihilist) do appear to cause trouble for both Simion's explanation proffering and my understanding norm anyway ${ }^{15}$

I agree that this is a problem for both Simion and myself. That said, it is not a problem that is distinctive to moral assertion. In fact, it is well known problem from the literature on the knowledge norm of assertion. The good news is that the literature features a variety of responses that are available for both Simion and myself (e.g. Milic 2017; Sosa 2010; Turri

\footnotetext{
${ }^{15}$ That the moral nihilist violates the understanding norm of moral assertion follows straightforwardly on some accounts of understanding, including the received view according to which understanding why $p$ just is knowledge why p (e.g. Lipton 2004). It may be less clear on other views. Perhaps it might even be thought that the case of the moral nihilist shouldn't be a problem for the understanding norm since it's independently plausible that the nihilist has the relevant moral understanding. Be that as it may, it is easy to that there are cases in the vicinity that do cause trouble for the understanding view. Consider, for instance, a child who has been told that they oughtn't buy from the corner shop because the owner is a racist. When parroting what they have been told, their assertions are intuitively fine. However, since they are simply parroting what they have been told, they don't have the relevant moral understanding.
} 
2016). I will here outline my own preferred solution (Kelp 2018) which draws once more on the normative framework associated with e-functional types developed above.

Recall that e-functions have normative import. In particular, for e-functional types, there are evaluative norms and prescriptive norms. Evaluative norms concern what is good. In the case of functional types, the evaluative norm has it that a token of a functional type is good if and only if it has the disposition to fulfil its e-function when functioning normally in normal conditions. Prescriptive norms concern ought to do-s. In the case of functional types, they specify how various parts of the system ought to work as follows: a part of the system functions as it ought to if and only if it functions normally.

Note that evaluative and prescriptive norms can come apart. Most importantly for present purposes, a token of a functional type may satisfy the evaluative norm without the producer of the token satisfying the prescriptive norm governing the production of the token. By way of illustration, let's return to the coffee case once more. Recall, first, that a good cup of coffee is one that has the disposition to generate pleasant gustatory experiences in consumers when functioning normally in normal conditions. Now suppose that the coffee shop operates a prescriptive norm for how to make coffee: a certain procedure that the barista needs to follow (say because following this procedure is what has contributed to keeping the coffee shop alive and so counts as normal functioning). Crucially, it may be that this prescriptive norm is broken and yet a perfectly good cup of coffee is produced. The prescriptive norm is broken because the barista deviated from the procedure. At the same time, the cup of coffee produced is good in virtue of the fact that it has the disposition to generate pleasant gustatory experiences in hearers when functioning normally in normal conditions. It is thus possible for a token of a functional type to satisfy the evaluative norm for functional items even though it was not produced in compliance with the prescriptive norm for production governing the producer. ${ }^{16}$

Crucially, in a similar way, in cases of selfless moral assertions the evaluative norm of moral assertion is satisfied while the prescriptive norm isn't. For instance, our nihilist violates the prescriptive norm of moral assertion because she doesn't believe and hence does know/understand what she asserts. At the same time, her assertion satisfies the evaluative norm for moral assertion. After all, it has the disposition to generate moral understanding in hearers when functioning normally in normal conditions.

It is now easy to see how the intuition that selfless moral assertions are fine can be accommodated. Selfless moral assertions are good assertion and that's what the intuition picks up on.

12. Recall that, on my view, to live up to the norm of moral assertion, speakers must not only possess understanding why, they must also offer explained moral assertions. This allows my view to avoid Simion's worry that understanding why alone is not sufficient for permissible moral assertion. At the same time, one might wonder whether the claim that proper moral assertion needs to be explained isn't too strong. In fact, in

\footnotetext{
${ }^{16}$ It is now easy to see just why it is that the tokens that need to be functioning normally, not the entire system (fn.9). As the above case clearly indicates, it is possible that a producer of a token (and hence the entire system) may not be functioning normally, even though the token produced is a perfectly good token of its type.
} 
recent work, Max Lewis (2019) presses exactly this worry. He offers two cases of proper moral assertion without explanation. Here goes:

Moral Advice: Alex and Ben are good friends. Ben sees Alex's boyfriend, Carl, kissing another person at a nightclub. Ben is unsure of what to do. Finding out might crush Alex and destroy an otherwise healthy relationship. Ben asks his friend Denise what to do. Denise thinks about the case and particular moral issues at play. It strikes Denise as clear that Ben ought to inform Alex. Denise asserts, "The morally best thing to do in this case is to tell Alex what you saw." Ben thanks Denise for her advice. (Lewis 2019, 1047).

Antebellum Slavery: Michael is the son of a slave owner in the antebellum south of the United States. He has lived on the same estate as slaves his entire life. Now 21, Michael's long-lived doubts about the morality of slavery have come to a head and he now knows that slavery is wrong and he also grasps why. During an argument with his father, he says, "How could you be so blind? Slavery is clearly wrong!" (Lewis 2019, 1047).

In both cases, speakers make intuitively proper moral assertions without also offering explanations. Doesn't this mean that any norm of moral assertion that requires explanations is too strong?

No. To see why not, note first that, in Moral Advice, Denise's utterance of "The morally best thing to do in this case is to tell Alex what you saw." is not made in a void. On the contrary, it is clear from context that Ben is unsure what to do because he is unsure about which of two conflicting moral considerations takes precedence. And that's an important part of Denise's advice. What she is advising Ben to do is to tell Alex what he saw because disclosing this kind of information to Alex takes precedence over the attempt to protect Alex and their relationship. Similarly, the natural way to fill in the blanks of Antebellum Slavery is that there is a wide range of reasons why slavery is wrong present at the context of their conversation. While Michael says "Slavery is clearly wrong!" there can be no question that what he means is that it is clearly wrong for all the reasons present at their context. While these cases do indeed not feature explicit explanations, it doesn't follow that they don't qualify as explained moral assertions. Rather, what they highlight is that explanations can and often enough are given by context.

13. This paper has developed a puzzle about moral assertion. In particular, it has shown that three individually plausible theses, MAP1-3, cannot all be true. I have also provided reason to think that MAP2 and 3 can be exonerated, which put considerable pressure on the remaining suspect, i.e. MAP1, that assertion from knowledge is proper in default cases of moral assertion. Fortunately, it turns out that there is independent reason to think that MAP1 is the culprit. While knowledge takes centre stage in the normativity of non-moral assertion, the same does not hold for the moral case. Rather, what's at the heart of the normativity of moral assertion is understanding why. The reason for this difference is ultimately rooted in a different in efunction of non-moral vs. moral assertion. While in case of non-moral assertion the e-function is to generate knowledge, in case of moral assertion it consists in generating moral understanding. From these two different epistemic e-functions we can derive different epistemic norms, both evaluative and prescriptive: in the case of non-moral assertion these norms feature knowledge and in the case of moral understanding they feature understanding. The Moral Assertion Puzzle thus receives an attractive solution. 
Open Access This article is licensed under a Creative Commons Attribution 4.0 International License, which permits use, sharing, adaptation, distribution and reproduction in any medium or format, as long as you give appropriate credit to the original author(s) and the source, provide a link to the Creative Commons licence, and indicate if changes were made. The images or other third party material in this article are included in the article's Creative Commons licence, unless indicated otherwise in a credit line to the material. If material is not included in the article's Creative Commons licence and your intended use is not permitted by statutory regulation or exceeds the permitted use, you will need to obtain permission directly from the copyright holder. To view a copy of this licence, visit http://creativecommons.org/licenses/by/4.0/.

\section{References}

Benton M (2011) Two more for the knowledge account of assertion. Analysis 71:684-687

Buller DJ (1998) Etiological theories of function: a geographical survey. Biol Philos 13:505-527

Burge T (1993) Content preservation. The Philosophical Review 102:457-488

Callahan L (2018) Moral testimony: a re-conceived understanding explanation. Philos Q 68:437-459

Coady C (1992) Testimony: a philosophical study. Clarendon Press, Oxford

Godfrey-Smith P (1994) A modern history theory of functions. Noûs 28:344-362

Goldberg S (2006) Reductionism and the distinctiveness of testimonial knowledge. In: Lackey J, Sosa E (eds)

The epistemology of testimony. Oxford University Press, Oxford

Goldman A (1999) Knowledge in a social world. Clarendon Press, Oxford

Graham P (2010) Testimonial entitlement and the function of comprehension. In: Haddock A, Millar A, Pritchard

D (eds) Social epistemology. Oxford University Press, New York

Graham P (2012) Epistemic Entitlement. Noûs 46:449-482

Graham P (2014) Functions, warrant, history. In: Fairweather A, Flanagan O (eds) Naturalizing Epistemic Virtue.

Cambridge University Press, Cambridge

Griffith P (1993) Functional analysis and proper functions. Br J Philos Sci 44:409-422

Hawthorne J (2004) Knowledge and lotteries. Oxford University Press, Oxford

Hills A (2009) Moral testimony and moral epistemology. Ethics 120:94-127

Howard N (2018) Sentimentalism about moral understanding. Ethical Theory Moral Pract 21:1065-1078

Kelp C (2015) Understanding phenomena. Synthese 192:3799-3816

Kelp C (2018) Assertion: a Function first account. Noûs 52:411-42

Kelp C (2020) Moral understanding. (unpublished)

Lackey J (2008) Learning from words: testimony as a source of knowledge. Oxford University Press, Oxford Lewis M (2019) The norm of moral assertion: a reply to Simion. Ethical Theory Moral Pract 22:1043-1049

Lewis M (2020) Moral deference, moral assertion, and pragmatics. Ethical Theory and Moral Practice 23:5-22 Lipton P (2004) Inference to the best explanation. Routledge, London and New York

McGrath S (2011) Skepticism about moral expertise as a puzzle for moral realism. J Philos 108:111-137

Milic I (2017) Against selfless assertion. Philos Stud 174:2277-2295

Millikan R (1984) Language, thought, and other biological categories. MIT Press, Cambridge/MA

Millikan R (2004) Varieties of meaning. MIT Press, Cambridge/MA

Neander K (1991) The teleological notion of `function. Australas J Philos 69:454-468

Nickel P (2001) Moral testimony and its authority. Ethical Theory Moral Pract 4:253-266

Simion M (2016) Assertion: knowledge is enough. Synthese 193:3041-3056

Simion M (2018) The explanation proffering norm of moral assertion. Ethical Theory Moral Pract 21:477-488

Simion M (2019) Knowledge first functionalism. Philosophical Issues 29:254-267

Simion M, Kelp C (2020) How to be an anti-reductionist. Synthese 197, 2849-66

Sliwa P (2017) Moral understanding as knowing right from wrong. Ethics 127:521-552

Sosa E (2010) Value matters in epistemology. J Philos 107:167-190

Turri J (2011) The express knowledge account of assertion. Australas J Philos 89:37-45

Turri J (2015) Understanding and the norm of explanation. Philosophia 43:1171-1175

Turri J (2016) Knowledge and the norm of assertion: an essay in philosophical science. Open Book, Cambridge Unger P (1975) Ignorance: a case for Scepticism. Clarendon, Oxford

Williamson T (2000) Knowledge and its limits. Oxford University Press, Oxford

Wolff, R. 1970. In defense of anarchism. New York/NY

Publisher's Note Springer Nature remains neutral with regard to jurisdictional claims in published maps and institutional affiliations. 